Revista Brasileira de Agricultura Irrigada v.14, no .4, p. 4162 - 4170, 2020

ISSN 1982-7679 (On-line)

Fortaleza, CE, INOVAGRI - http://www.inovagri.org.br

DOI: $10.7127 /$ rbai.v14n401188

Protocolo 1188.20 - 07/09/2020 Aprovado em 02/02/2021

\title{
IRRIGAÇÃO E COBERTURAS DE SOLO NA PRODUÇÃO DA PIMENTEIRA ARDIDA NO SUDESTE BRASILEIRO
}

\author{
Jonathan dos Santos Viana ${ }^{1}$, Luiz Fabiano Palaretti ${ }^{2}$, Gustavo Michalski Tessaro ${ }^{3}$, Júlia Ramos \\ Guerreiro $^{4}$, Rogério Teixeira de Faria ${ }^{5}$
}

\begin{abstract}
RESUMO
A pimenta dedo de moça é uma olerícola importante na agricultura brasileira, no entanto, é necessário o manejo adequado da irrigação. Diante deste contexto, objetivou-se com este trabalho avaliar o efeito de diferentes níveis de irrigação com e sem o uso de mulching nos componentes de produção da pimenteira dedo de moça. O experimento foi desenvolvido em cultivo protegido, na Unesp - Câmpus Jaboticabal, SP, Brasil. Empregou-se o delineamento experimental em blocos inteiramente casualizados, com seis repetições. Os tratamentos consistiram em T1: nível de irrigação 50\% CV + solo sem cobertura; T2: nível de irrigação 50\% CV + mulching branco; T3: nível de irrigação 100\% CV + solo sem cobertura; T4: nível de irrigação 100\% CV + mulching branco. Avaliou-se altura de planta, diâmetro do caule, massa fresca da parte aérea, número de frutos e a produção. $\mathrm{O}$ tratamento T3 influenciou diretamente na altura de planta $\left(96,81 \mathrm{~cm}^{-1}\right)$. Já para diâmetro do caule e matéria fresca parte aérea, os tratamentos T2, T3 e T4 não diferiram entre si. Maiores números de frutos e produções foram observadas para os tratamentos T3 e T4, de 36,33 e 45,67 frutos;120,41 g e 233,66 g.planta ${ }^{-1}$, respectivamente. A utilização do nível de irrigação $100 \% \mathrm{CV}$ associado com o uso de mulching branco influenciou positivamente em maior diâmetro do caule, matéria fresca parte aérea, número de frutos e produção da pimenteira dedo de moça em ambiente protegido.
\end{abstract}

Palavras-chave: Capsicum baccatum L., manejo de irrigação, proteção do solo.

\footnotetext{
${ }^{1}$ Doutorando em Agronomia (Ciência do Solo), Departamento de Engenharia e Ciências Exatas, UNESP - Jaboticabal. CEP: 14884-900, Via de Acesso Professor Paulo Donato Castelane S/N - Vila Industrial, Jaboticabal - SP, Brasil. Email: jonathan.viana@unesp.br

${ }^{2}$ Prof. Dr, Departamento de Engenharia e Ciências Exatas, UNESP - Jaboticabal. CEP: 14884-900, Via de Acesso Professor Paulo Donato Castelane S/N - Vila Industrial, Jaboticabal - SP, Brasil. Email: lfpalaretti@ fcav.unesp.br

${ }^{3}$ Graduando em Engenharia Agronômica, Departamento de Engenharia e Ciências Exatas, UNESP - Jaboticabal. CEP: 14884-900, Via de Acesso Professor Paulo Donato Castelane S/N - Vila Industrial, Jaboticabal - SP, Brasil. Email gumichalski@gmail.com

${ }^{4}$ Graduanda em Engenharia Agronômica, Departamento de Engenharia e Ciências Exatas, UNESP - Jaboticabal. CEP: 14884-900, Via de Acesso Professor Paulo Donato Castelane S/N - Vila Industrial, Jaboticabal - SP, Brasil. Email: julia.agro018@gmail.com

5 Prof. Dr, Departamento de Engenharia e Ciências Exatas, UNESP - Jaboticabal. CEP: 14884-900, Via de Acesso Professor Paulo Donato Castelane S/N - Vila Industrial, Jaboticabal - SP, Brasil. Email: rogeriofaria@fcav.unesp.br
} 


\title{
IRRIGATION LEVELS ASSOCIATED WITH SOIL COVERAGE IN THE PRODUCTION OF BURNED PEPPER IN SOUTHEAST BRAZIL
}

\begin{abstract}
The red pepper is an important vegetable in Brazilian agriculture, in view of its wide culinary use. For the studied culture to develop satisfactorily, adequate irrigation management and verification of its behavior using plastic mulching is necessary. Based on the importance of this culture, the objective of this work was to evaluate the effect of levels of irrigation and mulching on the production components of the pepper girl finger. The experiment was developed in protected cultivation, at Unesp - Câmpus Jaboticabal, SP, Brazil. A completely randomized block design with five replications was used. Each pot consisted of an experimental plot, where the treatments were installed. The treatments consisted of T1: irrigation level $50 \% \mathrm{CV}+$ bare soil; $\mathrm{T} 2$ : level of irrigation $50 \% \mathrm{CV}+$ white mulching; T3: level of irrigation $100 \% \mathrm{CV}+$ bare soil; T4: level of irrigation $100 \% \mathrm{CV}+$ white mulching. Plant height, stem diameter, fresh shoot weight and production per plant were evaluated. The T3 treatment directly influenced the plant height $(96.81 \mathrm{~cm}-1)$. For stem diameter and shoot fresh matter, treatments T2, T3 and T4 did not differ. Higher numbers of fruits and yields were observed for treatments T3 and T4, of 36.33 and 45.67 fruits, $120.41 \mathrm{~g}^{-1}$ and 233.66 g.plant $^{-1}$, respectively. The use of the $100 \% \mathrm{CV}$ irrigation level associated with the use of white mulching positively influenced a larger stem diameter, fresh shoot, number of fruits and production of the girl's finger pepper in a protected environment.
\end{abstract}

Keywords: Capsicum baccatum, irrigation management, mulching, protected cultivation.

\section{INTRODUÇÃO}

O setor hortícola continua em franca expansão, uma vez que a preferência alimentar sofreu modificações ao longo dos anos. Dentre as hortaliças, a pimenta (Capsicum spp.) tem grande destaque por sua versatilidade tanto para consumo quanto para o aproveitamento no setor industrial (FURLAN et al., 2015).

A pimenta dedo-de-moça (Capsicum baccatum var. pendulum) conhecida como pimenta-vermelha, calabresa ou chifre-deveado, é uma das mais consumidas no Brasil, principalmente nos estados do Rio Grande do Sul, São Paulo e Goiás. O seu cultivo é realizado por pequenos, médios e grandes produtores e se ajusta perfeitamente aos modelos de agricultura familiar e de integração pequeno agricultor-agroindústrias (CARVALHO et al., 2009). Pertencente à família Solanaceae e originária da América do Sul, a pimenta provavelmente foi um dos primeiros aditivos alimentares utilizados pelas civilizações antigas para conferir aroma, cor e o sabor aos alimentos (CRISÓSTOMO et al., 2006).
No Brasil a cultura apresenta elevada importância socioeconômica, pois promove a geração de emprego, além de estimular a agricultura familiar aumentando a renda de pequenos produtores (VILELA, 2004) e para pequenas indústrias de conservas.

Para incrementar o potencial produtivo da pimenteira dedo de moça estudos sob níveis de irrigação e coberturas no solo em ambiente protegidos são essenciais. Reis et al. (2013) relatam que o propósito do cultivo em ambiente protegido é melhorar a produtividade e a qualidade dos produtos agrícolas por oferecer regularidade na produção. Figueiredo (2011) concorda que esta modalidade de cultivo proporciona uma fuga da sazonalidade, fato que ocorre principalmente na olericultura, em função do clima. Essa fuga possibilita a obtenção de uma melhor remuneração da produção, pelo fato de a comercialização ser feita em um período com menor oferta e melhor preço.

A pimenteira é altamente sensível à falta de água no solo, como a maioria das hortaliças, principalmente durante o florescimento, a formação e o desenvolvimento dos frutos. A 


\section{IRRIGAÇÃO E COBERTURAS DE SOLO NA PRODUÇÃO DA PIMENTEIRA ARDIDA NO SUDESTE BRASILEIRO}

falta de água durante a floração pode reduzir a fixação e a qualidade dos frutos, assim como a produtividade. A insuficiência de água favorece também a queda e o abortamento de flores e frutos, a ocorrência de podridão apical e reduz o tamanho final dos frutos (REIFSCHNEIDER; RIBEIRO, 2008).

Por ser uma hortaliça fruto a exigência em termos gerais, varia de 500 a $800 \mathrm{~mm}$, podendo ultrapassar os $1.000 \mathrm{~mm}$ para cultivares de ciclo longo (EMBRAPA, 2007). $O$ manejo adequado da irrigação se faz necessário em regiões em que ocorre irregularidade nas precipitações, como é o caso da região Sudeste do Brasil, como também em ambientes protegidos onde o processo de evapotranspiração é maior quando comparado ao cultivo em campo.

Estudos de estratégias que viabilizem uma redução nas perdas de água por evaporação como também favoreça um melhor conforto térmico para a cultura da pimenteira dedo de moça, são primordiais.

Uma alternativa viável é o uso de mulching plástico que proporciona ao solo uma melhora na amplitude térmica, uma redução da evaporação de água, favorecendo a atividade microbiana, aumento nas taxas de mineralização da matéria orgânica e aumento de nitratos em camadas mais superficiais do solo (SAMPAIO; FONTES; SEDIYAMA, 1999).

Diante deste contexto, objetivou-se com este trabalho avaliar o efeito de diferentes níveis de irrigação com e sem o uso de mulching nos componentes de produção da pimenteira dedo de moça.

\section{MATERIAL E MÉTODOS}

O experimento foi conduzido no Setor de Plasticultura do Departamento de Engenharia e Ciências Exatas da FCAV/UNESP - Campus de Jaboticabal, latitude $21^{\circ} 15^{\prime} 22^{\prime}$ 'S e longitude $48^{\circ} 18^{\prime} 58^{\prime \prime}$ W, com altitude média de $595 \mathrm{~m}$. O clima da região, segundo classificação de Köppen, é do tipo Aw, tropical, seco no inverno e com chuvas no verão, apresentando temperatura média anual de $22,5^{\circ} \mathrm{C}$ (GARCIA; ANDRÉ, 2015).

Os dados climáticos de temperatura e umidade ocorridos durante a execução do trabalho estão apresentados nas Figuras 1.

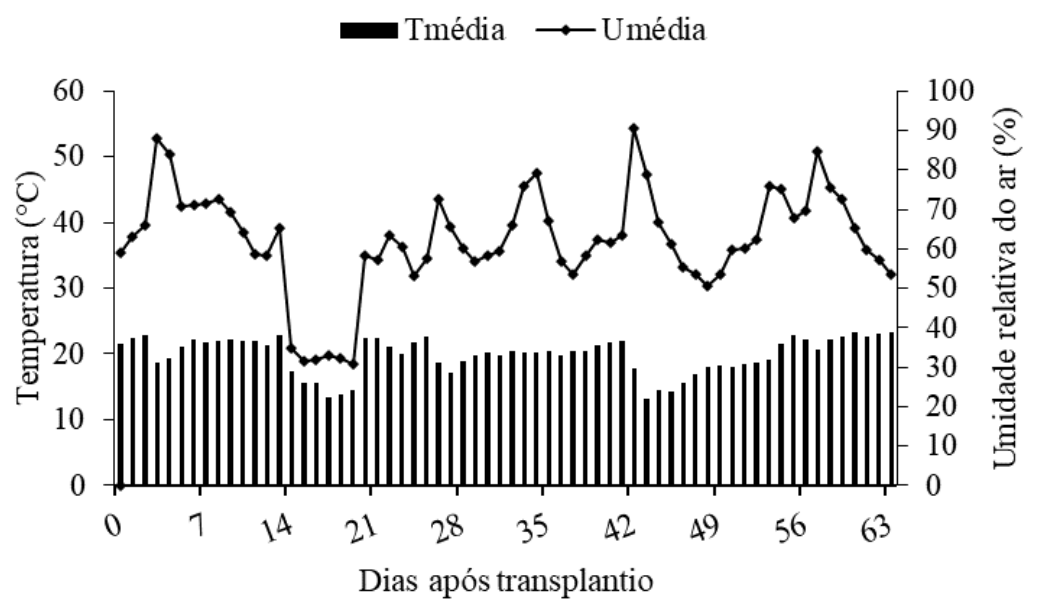

Figura 1. Variação de temperatura (Tmédia) e umidade relativa do ar (Umédia) em ambiente protegido. UNESP, Jaboticabal - SP, 2020.

Foram utilizados vasos de $12 \mathrm{dm}^{3}$ contendo solo classificado como Latossolo Vermelho distrófico típico (Latossolo), textura argilosa, horizonte A moderado, conteúdo de óxido de ferro $<8 \%$ e relação $\left(\mathrm{SiO}_{2}\right) /\left(\mathrm{Al}_{2} \mathrm{O}_{3}+\right.$ $\left.\mathrm{Fe}_{2} \mathrm{O}_{3}\right)>0,75$ (EMBRAPA, 2018) cujas características químicas estão contidas na Tabela 1. 
Viana et al.

Tabela 1. Análise de solo da área experimental. UNESP, Jaboticabal - SP, 2020.

\begin{tabular}{|c|c|c|c|c|c|c|c|c|c|c|c|}
\hline \multirow{2}{*}{$\mathrm{pH}$} & \multirow{2}{*}{ M.O. } & \multirow{2}{*}{$\mathrm{P}$} & \multirow{2}{*}{ S } & \multirow{2}{*}{$\mathrm{Ca}$} & \multirow{2}{*}{$\mathrm{Mg}$} & \multirow{2}{*}{$\mathrm{Na}$} & \multirow{2}{*}{ K } & \multirow{2}{*}{$\mathrm{Al}$} & $\mathrm{H}+\mathrm{Al}$ & \multirow{2}{*}{ S.B. } & \multirow{2}{*}{ CTC } \\
\hline & & & & & & & & & SMP & & \\
\hline $\mathrm{CaCl}_{2}$ & $\mathrm{~g} \mathrm{dm}^{-3}$ & \multicolumn{2}{|c|}{$\mathrm{mg} \mathrm{dm}^{-3}$} & \multicolumn{8}{|c|}{$\mathrm{mmol}_{\mathrm{c}} \mathrm{dm}^{-3}$} \\
\hline 6,3 & 24 & 52 & 11 & 30 & 9 & - & 2,3 & 0 & 17 & 41,0 & 57,8 \\
\hline
\end{tabular}

*Athenas: Consultoria e Laboratórios.

Com base no resultado da análise química do solo foi realizada a adubação de transplantio, conforme recomendação do Boletim 100 do IAC (VAN RAIJ et al ., 1997 ), para a cultura da pimenteira hortícola. Para tanto foram aplicados $40 \mathrm{~kg} \mathrm{ha}^{-1} \mathrm{de} \mathrm{N}$ na forma de ureia, $120 \mathrm{~kg} \mathrm{ha}^{-1}$ de $\mathrm{P}_{2} \mathrm{O}_{5}$ na forma de superfosfato simples e $320 \mathrm{~kg} \mathrm{ha}^{-1}$ de $\mathrm{K}_{2} \mathrm{O}$ na forma de cloreto de potássio. $\mathrm{Na}$ adubação de cobertura foram utilizados $80 \mathrm{~kg} \mathrm{ha}^{-1}$ de $\mathrm{N}$ e 80 $\mathrm{kg} \mathrm{ha}^{-1}$ de $\mathrm{K}_{2} \mathrm{O}$, parcelados em seis vezes.

Empregou-se o delineamento experimental em blocos inteiramente casualizados, com seis repetições. Os tratamentos consistiram em T1: nível de irrigação $50 \% \mathrm{CV}$ + solo sem cobertura; T2: nível de irrigação 50\% CV + mulching branco; T3: nível de irrigação $100 \% \mathrm{CV}+$ solo sem cobertura; T4: nível de irrigação $100 \%$ CV + mulching branco.

O nível de irrigação de $100 \%$ da capacidade de vaso foi obtido através da saturação solo no vaso e posterior drenagem, definindo assim a "capacidade de vaso", que corresponde a quantidade máxima de água que pode ser retida no volume de solo considerado (CASAROLI; JONG VAN LIER, 2008). As lâminas totais aplicadas durante condução do experimento variaram conforme os níveis de irrigação e presença e/ou ausência de mulching. Para tanto foram aplicados: 443,88 mm; 378 $\mathrm{mm} ; 846,94 \mathrm{~mm}$ e $764,29 \mathrm{~mm}$, referentes aos tratamentos: T1, T2, T3 e T4, respectivamente.

As avaliações foram efetuadas em cada unidade experimental, correspondendo a uma planta por vaso, mensurando os seguintes parâmetros: altura de plantas (AP, cm), diâmetro do caule (DC, $\mathrm{mm}$ ), massa fresca parte aérea (MFárea, g), número de frutos (NF, frutos planta ${ }^{-1}$ ) e produção (Prod, g planta ${ }^{-1}$ ).

Os dados foram submetidos à análise de variância pelo teste $\mathrm{F}(\mathrm{P}<0,05)$, e as médias foram comparadas pelo teste de Tukey, por meio do software Agroestat, versão 1.0 (BARBOSA ; MALDONADO JÚNIOR, 2015).

\section{RESULTADOS E DISCUSSÃO}

O resumo das análises de variância (Tabela 2) indica efeito significativo dos níveis de irrigação e cobertura no solo $(\mathrm{P}<0,01)$ na altura de plantas (AP) e diâmetro do caule (DC) aos 35 dias após transplantio (DAT) das mudas de pimenta, na massa fresca parte aérea (MFaérea), número de frutos (NF, frutos planta ${ }^{1}$ ) e produção (Prod, g planta ${ }^{-1}$ ) aos 63 DAT.

Tabela 1. Resumo das análises de variância da altura de plantas $(\mathrm{cm})$, diâmetro de caule $(\mathrm{mm})$, massa fresca parte aérea $\left(\mathrm{g}\right.$ planta $\left.^{-1}\right)$, número de frutos (frutos planta $\left.{ }^{-1}\right)$ e produção $\left(\mathrm{g} \mathrm{planta}^{-1}\right)$, em função de níveis de irrigação e cobertura no solo para cultura da pimenteira dedo de moça em ambiente protegido.

\begin{tabular}{|c|c|c|c|c|}
\hline \multirow[b]{2}{*}{ Parâmetros avaliados } & \multicolumn{2}{|c|}{ Fontes de variação } & \multirow[b]{2}{*}{$\mathrm{CV}(\%)$} & \multirow[b]{2}{*}{ Média geral } \\
\hline & Tratamentos & Bloco & & \\
\hline Altura de plantas & $8,34 * *$ & $0,42^{\mathrm{ns}}$ & 33,85 & 79,97 \\
\hline Diâmetro do caule & $4,63 * *$ & $0,51^{\mathrm{ns}}$ & 8,55 & 10,50 \\
\hline Matéria fresca parte aérea & $7,21 * *$ & $0,45^{\mathrm{ns}}$ & 19,35 & 137,92 \\
\hline Número de folhas & $13,81 * *$ & $2,34^{\mathrm{ns}}$ & 28,60 & 27,25 \\
\hline Produção & $16,03^{* *}$ & $0,97^{\mathrm{ns}}$ & 23,49 & 110,42 \\
\hline
\end{tabular}

**Significativo a 1\% pelo teste F; *Significativo a 5\% pelo Teste F; ns - Não significativo pelo teste F; CV (\%): Coeficiente de variação. 
IRRIGAÇÃO E COBERTURAS DE SOLO NA PRODUÇÃO DA PIMENTEIRA ARDIDA NO SUDESTE BRASILEIRO

Na Figura 2 encontram-se os valores médios para as variáveis altura de plantas e diâmetro do caule para pimenteira dedo de moça submetida a níveis de irrigação e cobertura do solo em ambiente protegido. Para as variáveis supracitadas foram verificadas diferenças significativas $(\mathrm{P}<0,05)$.
Aos 63 DAT, a maior altura de planta estimada foi de $96,81 \mathrm{~cm}$ (T3), superior $92,8 \%$ em relação à altura $75,21 \mathrm{~cm}$ (T4). Já para diâmetro do caule maior valor encontrado foi de 11,44 $\mathrm{mm}$ (T4), superior 80,6 \% em relação ao diâmetro 9,23 $\mathrm{mm}(\mathrm{T} 1)$.
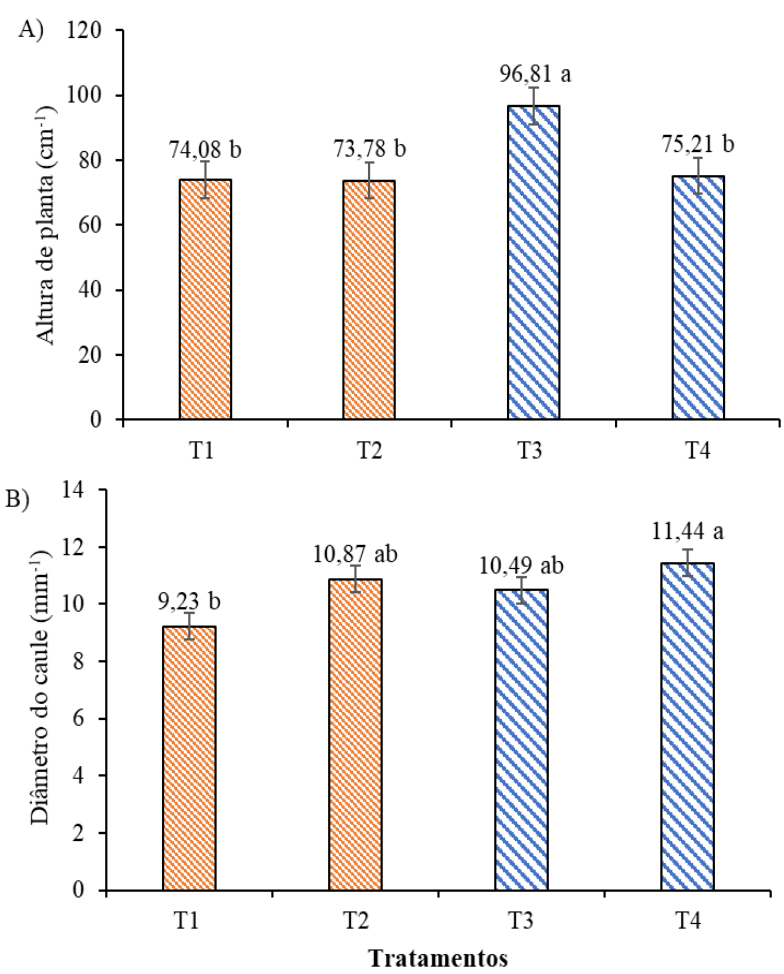

Figura 2. Valores médios estimados de altura de plantas (A) e diâmetro do caule (B) para pimenteira dedo de moça sob níveis de irrigação e cobertura do solo em ambiente protegido. T1: nível de irrigação $50 \% \mathrm{CV}$ + solo sem cobertura; T2: nível de irrigação 50\% CV + mulching branco; T3: nível de irrigação 100\% CV + solo sem cobertura; T4: nível de irrigação $100 \% \mathrm{CV}+$ mulching branco.

Para altura de plantas (Figura 2A) o tratamento T3 teve melhor representatividade, isso se deve à maior demanda hídrica da cultura, devido o solo está descoberto, fazendo com que a à evapotranspiração da cultura aumentasse. Esse aumento em altura de planta favorece maior emissão de ramos laterais, como também influencia diretamente em maior predominância de botais florais, associado a uma melhor atividade fotossintética. Resultados obtidos por Rezende et al. (2002) demonstraram que a altura de planta da pimenteira dedo de moça foram influenciados pelo aumento de volume de água aplicado, corroborando com o comportamento obtido no presente trabalho. Resultados divergentes foram obtidos por Padrón et al. (2015), em pesquisa sobre lâminas e frequências de irrigação em pimentão cultivado em campo, ao constatarem menor altura de plantas, de 72,4 cm, no nível de irrigação de $100 \%$ da ETc, e maior altura, de $91,5 \mathrm{~cm}$, no nível de $60 \%$ da ETc, utilizando irrigação diária.

$\mathrm{O}$ menor diâmetro observado (Figura 2B) foi para o tratamento T1, nível 50\% CV + solo sem cobertura. Percebe-se para o tratamento T2 e T4, com e sem restrição hídrica e com a presença de mulching na cor branca por vaso, valores de $10,87 \mathrm{~mm}$ e $11,44 \mathrm{~mm}$. Mesmo com a restrição hídrica o comportamento para 
diâmetro do caule da pimenteira dedo de moça foram semelhantes estatisticamente. Isso se deve ao mulching na cor branca favorecer melhor ambiência e menores perdas por evaporação do solo nos vasos, o que acarretou de forma positiva em maiores diâmetros de caules. Maiores diâmetros de caules favorecem maior acúmulo de seiva pela planta, que impacta diretamente na produção de frutos de pimenta de boa qualidade. O mesmo comportamento foi encontrado por Paula (2008) onde se verificou incremento do diâmetro do caule de plantas de pimentão (Capsicum annuum L.) pertencente ao mesmo gênero da pimenta, em função do aumento do volume de água aplicado na irrigação, em cultivo em ambiente protegido, em PiracicabaSP.

Para as variáveis massa fresca da parte área (Figura $3 \mathrm{~A}$ ), número de frutos (Figura 3 B) e produção (Figura $3 \mathrm{C}$ ) também foram constatadas diferenças significativas $(\mathrm{P}<0,005)$.

Maiores massas frescas da parte aérea foram encontradas para os tratamentos $\mathrm{T} 2, \mathrm{~T} 3$, e T4 de 149,22; 146,98 e 157,67 g planta $^{-1}$, que diferiram do tratamento T1. Já para as variáveis número de frutos e produção da pimenteira dedo de moça maiores médias obtidas foram para os tratamentos T3 e T4.
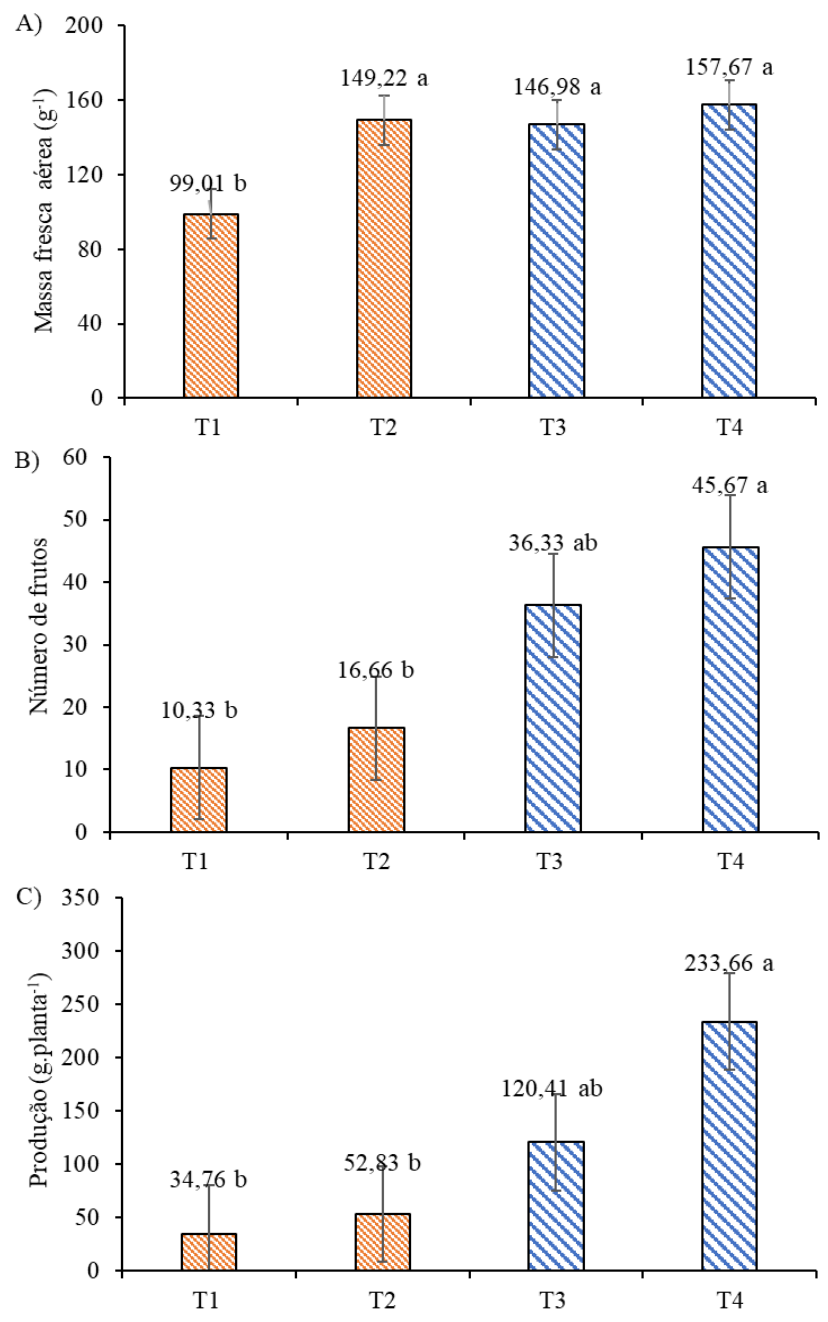

Figura 3. Valores médios estimados de massa fresca da parte aérea (Figura 3 A), número de frutos (Figura 3 B) e produção (Figura $3 \mathrm{C}$ ) para pimenteira dedo de moça sob níveis de irrigação e cobertura do solo em ambiente protegido. T1: nível de irrigação 50\% CV + solo sem cobertura; T2: nível de irrigação 50\% CV + mulching branco; T3: nível de irrigação 100\% CV + solo sem cobertura; T4: nível de irrigação $100 \%$ CV + mulching branco. 
A água influenciou diretamente no aumento de biomassa da parte aérea da pimenteira dedo de moça, com ou sem uso de cobertura (Figura 3 A). Esse incremento de água aplicada favorece em maior atividade fotossintética realizada pela planta que ocasiona aumento na captação de energia luminosa pelas folhas implicando em maior produção de fotoassimilados tendo papel importante nos componentes de produção da pimenteira.

Quando se utiliza o manejo adequado da água de irrigação em vaso sem que ocorra lavagem do solo, como dos nutrientes ali presentes, isso gera maior aproveitamento dos nutrientes como também da água, o que foi observado para as maiores massas frescas da parte aérea (Figura $3 \mathrm{~A}$ ).

O nível de irrigação $100 \%$ CV atrelado ao solo do vaso com ou sem cobertura utilizando-se mulching na cor branca (T3 e T4) tiveram impacto direto no aumento do número de frutos e produção da pimenteira. Com a utilização de restrição hídrica $(50 \% \mathrm{CV}) \mathrm{com}$ ou sem mulching cor branca ocorre uma menor produção de biomassa da parte aérea que reduz diretamente no número de frutos e produção da pimenteira dedo de moça.

O menor valor de matéria fresca da parte aérea obtido neste trabalho está em acordo com Marinho (2011) ao identificar que o déficit de irrigação em pimenta cv Tabasco afetou negativamente a produção intermediaria $\mathrm{e}$ tardia, resultando em menor produção total ou massa de frutos por planta. Lima et al. (2013) também relatam a tendência na redução de produtividade da pimenteira com aumento do déficit hídrico.

\section{CONCLUSÕES}

Os melhores resultados para altura de plantas da cultura da pimenteira dedo de moça foi obtido com a utilização do nível de irrigação correspondente a $100 \%$ da capacidade do vaso mais solo sem cobertura (T3).

A aplicação do menor nível de irrigação (50\% da capacidade do vaso), com ou sem o uso de mulching, propiciou reduções em todas as variáveis analisadas na cultura da pimenteira dedo de moça.

\section{AGRADECIMENTOS}

Os autores agradecem à Coordenação de Aperfeiçoamento de Pessoal de Nível Superior (CAPES - 001) pela concessão da bolsa que auxiliou na realização do presente estudo.

\section{REFERÊNCIAS}

BARBOSA, JC; MALDONADO JÚNIOR, W. 2015. Experimentação agronômica e agroestat - Sistemas para análises estatísticas de ensaios agronômicos. Jaboticabal: Multipress Ltda.

CASAROLI, D.; JONG VAN LIER, Q.D. 2008. Critérios para determinação da capacidade de vaso. Revista Brasileira de Ciência do Solo 32:59-66. https://doi.org/10.1590/S01000683200800010 0007.

CARVALHO, S.I.C.; RIBEIRO, C.S.C.; HENZ, G.P.; REIFSCHNEIDER, F.J.B. 2009. 'BRS Mari': nova cultivar de pimenta dedo-demoça para processamento. Horticultura Brasileira 27:571-573. https://dx.doi.org/10.1590/S010205362009000400028.

CRISÓSTOMO, J. R., FURTADO, R. F.; ABREU, F. R.; CRISOSTOMO, L. A.; MIRANDA, F. R.; BLEICHER, E.; RODRIGUES, S. M.; WEBER, O. B.; REIS, A.; ROCHA FILHO, R. R.; GONDIM, R. S.; GIRÃO, E. G. 2006. Cultivo de pimenta tabasco no Ceará. Fortaleza, Embrapa Agroindústria Tropical (Sistemas de Produção, 3), $40 \mathrm{p}$.

EMBRAPA - Empresa Brasileira de Pesquisa Agropecuária. 2018. Centro Nacional de Pesquisa de Solos. Sistema brasileiro de classificação de 
solos, 5.ed. Rio de Janeiro: Embrapa Solos. 590p.

EMBRAPA HORTALIÇAS. Irrigação de pimenteiras. 2007. Disponível em:< http://www.cnph.embrapa.br/paginas/serie.../p ublicacoes2007/ct_51.pdf >. Acesso em: $01 \mathrm{de}$ novembro de 2020 .

FURLAN, J.C.; SILVA JÚNIOR, R.L.S.; XAVIER, R.C.; NASCIMENTO, M.V.; FERNANDES, L.R.S.G.; BENETT, K.S.S. 2015. Produção de pimenta malagueta em função da adubação nitrogenada e do gel hidroretentor. In: XXXV Congresso Brasileiro de Ciência do Solo, 2015, Natal, RN: Centro de Convenções, 2015.

FIGUEIREDO, G. 2011. Panorama da Produção em Ambiente Protegido. Casa da Agricultura: Produção em Ambiente Protegido, Guararema, n. 2, p.10-12.

GARCIA, A., ANDRÉ, R.G.B. 2015. Variabilidade temporal da temperatura do ar em Jaboticabal - SP. Nucleus, v.12 n.1, p.181188. https://doi.org/10.37378/1982.2278.1443.

LIMA, E. M. C.; CARVALHO, J. A.; REZENDE, F. C.; THEBALDI, M.S.; GATTO, R. F. 2013. Rendimento da pimenta Cayenne em funcao de diferentes tensoes de água no solo. Revista Brasileira de Engenharia Agricola e Ambiental, v. 17, n.11, p. 1181-1187, Campina Grande. https://doi.org/10.1590/S14154366201300110 0008 .

MARINHO, L. B. Irrigacao plena e com deficit em pimenta cv Tabasco em ambiente protegido. Piracicaba, 2011.56p. Tese Doutorado.

PADRÓN, R. A. R.; RAMÍREZ, L. R.; CERQUERA, R.R.; NOGUEIRA, H.M.C.M.; MUJICA, J.L.U. Desenvolvimento vegetativo de pimentão cultivado com lâminas e frequências de irrigação. Tecnologia \& Ciência Agropecuária, v. 9, n. 2, p. 49-55, 2015. DOI: https://zeoserver.pb.gov.br/gestao- unificada/gu/emepa/publicacoes/revistatcaeme pa/edicoes/volume-09-2015/volume-9numero2-abril-2015/tca9209.pdf

PAULA, F.L.M. 2008. Aplicação de $\mathbf{C O}_{2}$ via irrigação na pimenta tabasco cultivada em ambiente protegido. 133 p. Tese (Doutorado em Irrigação e Drenagem) Escola Superior de Agricultura "Luiz de Queiroz “, Universidade de São Paulo, Piracicaba, 2008.

REIIFSCHNEIDER, F. J. B. 2000. Capsicum Pimentas e Pimentões do Brasil. Brasília: Embrapa comunicação para transferência de tecnologia/ Embrapa Hortaliças, 113p.

REIS, L. S.; AZEVEDO, C. A. V. de; ALBUQUERQUE, A. W.; JÚNIOR, J. F. S. 2013. Índice de área foliar e produtividade do tomate sob condições de ambiente protegido. Revista Brasileira de Engenharia Agrícola e Ambiental, p.386-391.

REIFSCHNEIDER, F. J. B.; RIBEIRO, C. S. C. 2008. Cultivo. In: Ribeiro, C.S.C.; Carvalho, S.I.C.; Henz, G. P.; Reifschneider, F.J.B. Pimentas Capsicum. Brasília: Embrapa Hortaliças, p.11-14.

REZENDE, F.C.; FRIZZONE, J.A.; BOTREL, T.A.; PEREIRA, A.S. 2002. Plantas de pimentão cultivadas em ambiente enriquecido com $\mathrm{CO}_{2}$. I. Crescimento vegetativo. Acta Scientarium Agronomy, Maringá, v.24, n.5, p. 1517-1526.

SAMPAIO, R.A.; FONTES, P.C.R.; SEDIYAMA, C.S. 1999. Resposta do tomateiro à fertirrigação potássica e cobertura do solo. Pesquisa Agropecuária Brasileira, Brasilia, v. 34, n.1, p.21-30.

VILELA, N.J. 2004. Coeficientes técnicos, custos, rendimento e rentabilidade. In: LOPES, A.C.; RIBEIRO, C.S.C.; CRUZ, D.M.R.; $\quad$ FRANÇA, F.H.; REIFSCHNEIDER, F.J.B.; HENZ, J.P.; SILVA, H.R.; CARVALHO, S.I.A.; MAROUELLI, W.A. 
SUDESTE BRASILEIRO

PEREIRA, W. Sistema de produção de pimentas (Capsicum spp.), Brasilia: Embrapa Hortaliças.
VAN RAIJ, B; CANTARELLA, H; QUAGGIO, JÁ; FURLANI, AMC. 1997. Boletim 100: Recomendações de adubação e calagem para o Estado de São Paulo, 2.ed. Campinas: Instituto Agronômico / Fundação IAC, 173p. 